\title{
Sprachwissenschaft
}

\section{Teuta Abrashi}

Universität Prishtina, Prishtina

DOI: $10.19195 / 0435-5865.142 .7$

\section{Zu syntaktischen Eigenheiten des Merkmals Person im Deutschen}

Wir begegnen dem grammatischen Merkmal Person sowohl in der nominalen als auch in der verbalen Domäne sprachlicher Einheiten. Der vorliegende Beitrag untersucht, in welchem Maße das Merkmal Person in der einen und in der anderen Domäne vertreten ist und wie seine Vertretung in beiden Bereichen reflektiert wird. Obwohl die Kategorie Person zu den grundlegenden grammatischen Kategorien gehört und der Eindruck besteht, dass alles darüber bereits ausgesagt wurde, sieht es aber tatsächlich so aus, dass besonders in der nominalen Domäne einige Gegebenheiten noch genauer zu erörtern sind. Aus diesem Grunde stelle ich im ersten Abschnitt des Beitrages zunächst einige grundlegende Kenntnisse über das Merkmal Person dar. Ich untersuche dann im zweiten Abschnitt das Merkmal Person im nominalen Bereich und versuche eine Antwort auf die Frage zu geben, ob Nomen über noch ein anderes Merkmal als das der 3. Person verfügen. Wie die Ausführungen zeigen werden, ist das der Fall. Deshalb sehe ich für den 3. Abschnitt des Beitrages einen Test bezüglich der die Auswirkung des ermittelten Untersuchungsbefundes auf Gegebenheiten, die Sprachbeschreibungen zu berücksichtigen haben, vor. Der Test wird an den syntaktischen Relationsverfahren der Kongruenz und Rektion vorgenommen. Aus ihm erwächst in einem weiteren Schritt ein Vorschlag, um eventuelle Schwierigkeiten zu überwinden. Meine zusammenfassenden Abschlussbemerkungen verfolgen letztlich das Ziel, die gewonnenen Untersuchungsergebnisse zu verallgemeinern, um auf diese Weise auch weitergehende sprachwissenschaftliche Diskussionen anzuregen.

\section{Das Merkmal Person - grundlegende Kenntnisse}

Traditionell wird angenommen, dass das Merkmal Person die Teilnehmer (Partizipanten) oder Nicht-Teilnehmer (Nicht-Partizipanten) in einer Sprachsituation 
kodiert. Die kognitive Grundlage des Merkmals Person ist somit bekanntlich von der Grundstruktur einer Sprechhandlung abgeleitet, es verkörpert einen Moment der Beziehung zum Sprechakt und zu seinen Teilnehmern (der Sprecher, Hörer und die abwesende Person). ${ }^{1}$ Dementsprechend unterscheiden Beschreibungen der deutschen und auch anderen Sprachen drei Personen: 1. (ich), 2. (du) und 3. Person (er, sie, es) bzw. den Sprecher, Angesprochenen und die besprochene Person. ${ }^{2}$

(1)

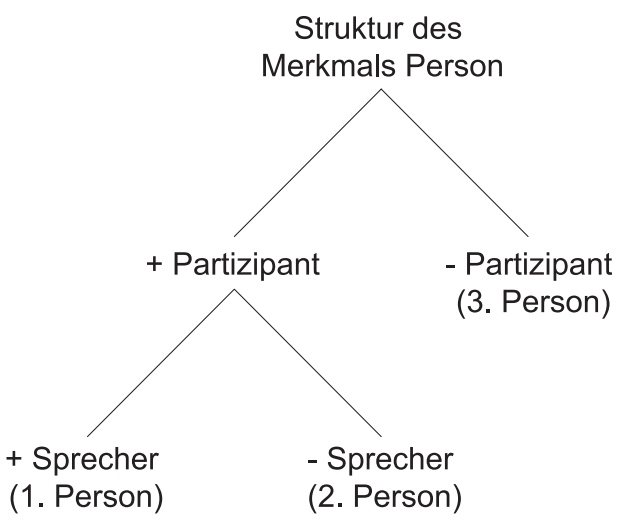

Zwischen 1./ 2. Person einerseits und der 3. Person anderseits besteht ein asymmetrischer struktureller Unterschied. ${ }^{3}$ In (1) sind die 1./ 2. Person ([+/- Sprecher]) nur für die Teilnehmer ([+ Partizipant]), während die 3. Person nur für die nicht-Teilnehmer.[- Partizipant] berechtigt. ${ }^{4}$ Ein anderer oppositioneller (struktureller) Unterschied des Merkmals Person kann den deiktischen Merkmalen zugeordnet werden: $\mathrm{ICH}[+$ ego; -tu] und nicht-ICH [-ego; +tu], wobei zur 1. Person das deiktische Merkmal [+ego; -tu] und 2./ 3. Person das deiktische Merkmal [-ego; $+\mathrm{tu}] /$ [-ego;-tu] gehören. ${ }^{5}$ Anders ausgedrückt, die Beziehung zwischen Teilnehmer und dem Redemoment kann ebenfalls durch deiktische Eigenschaften der

${ }^{1}$ Siehe z.B. Benveniste (1974).

2 Nicht alle Sprachen verfügen über die gleiche Anzahl von Komponenten der grammatischen Kategorie Person. Es gibt Sprachen mit 4., 5. und sogar mehr, aber auch Sprachen mit weniger als zwei Personen. Für einen typologischen Überblick vgl. Siewierska (2004).

3 Über asymmetrische strukturelle Unterschiede und (hierarchische) Ordnung der Personen siehe Plank (1985) und Corbett (2012: 20).

${ }^{4}$ Nach Heidolph et al. (1981: 540) ist aber die 3. Person hinsichtlich des Merkmals: ,persönliche Beteiligung am Sprechakt" als indifferent aufzufassen. In diesem Sinne wird in der 3. Person nicht angezeigt, ob eine Beteiligung am Sprechakt vorliegt. Wie die folgenden Beispiele zeigen, wird sie nicht ausgeschlossen (der Minister ordnet an ... =, ich ordne an'; man hat sich geirrt ... =, du hast dich geirrt'). Dagegen zeigen 1. und 2. Person an, dass eine persönliche Beteiligung am Sprechakt gegeben ist.

5 Demensprechend sieht die Struktur der deutschen Singularpersonalpronomina aufgrund der deiktischen Merkmalkomponenten folgendermaßen aus: ich $=[+$ ego; -du $], d u=[-$ ego; +du $]$ und er/ sie/es $=[-\mathrm{ego} ;-\mathrm{du}]($ Lyon 1980: 282). 
Kategorie Person dargestellt werden. ${ }^{6}$ In diesem Sinne kann die Bedeutung des Merkmals Person teilweise von der Perspektive der redenden Person abhängen. Wenn man sich einig ist, dass die erste Person der Redende selbst ist, die zweite Person sein Redepartner und zur dritten Person alle übrigen Lebewesen und Dinge gehören, dann ist der Standpunkt des Sprechers für die Wahl der Personenkategorien ausschlaggebend. Solange der Redende und der Redepartner involviert sind, handelt es sich eindeutig um deiktische Kategorien. Die Personen sind aber nicht immer deiktisch, so dass sich der Bezug zum Redemoment verliert, sobald von übrigen Lebewesen und Dingen die Rede ist. Demzufolge spielt jetzt die 3. Person eine Sonderrolle. ${ }^{7}$ Durch ihre logisch-grammatische Relation wird diese Wirklichkeit lediglich wiedergespiegelt, ohne jedoch einen Bezug zur Redesituation herzustellen. ${ }^{8}$

Wo liegt eigentlich der Ursprung der grammatischen Kategorie Person? Um diese Quelle zu finden, befassen wir uns zuerst mit der Kategorie Numerus und nehmen für diesen Zweck folgendes Beispiel:

\section{(2) Die Sterne leuchten.}

In (2) markieren sowohl das Subjekt als auch das Verb den Numerus. Diese Markierungen sind aber nicht gleichwertig. Die Sterne stehen aus semantischen Gründen im Plural und falls wir auf den Singular umschalten, würde dieses ebenso eine semantische Wahl darstellen. Auf der anderen Seite ist die Anwesenheit des Plurals an leuchten nicht semantisch motiviert, da nicht unbedingt mehr als ein leuchtendes Ereignis notwendig sein kann. Viel mehr deutet es auf eine syntaktische Motivation hin: der Numerus an leuchten hängt vom Numerus seines Subjekts ab. Dementsprechend können wir behaupten, dass die Markierung des Numerus im Nominalelement (die Sterne) gegeben ist, während es beim Verb (leuchten) zutrifft, aber nicht gegeben ist. Deswegen sagt man, dass der Numerus für die Sterne ein inhärentes und für leuchten „ein erzwungenes“ bzw. kontextuelles Merkmal ist.

Ähnliche Verhältnisse herrschen auch bei der Person. Es gibt Sätze im Deutschen, die über kein grammatisches Subjekt verfügen und trotzdem ihren kommunikativen Zweck erreichen:

(3) Iss! Schreib!

${ }^{6}$ Admoni (1982: 5).

${ }^{7}$ Nach Harweg (1968: 24) zeigt sich die Sonderrolle der 3. Person auch dadurch, dass die 3. Person (und die korrespondierten Pronomina) als Hersteller bestimmter Bezüge zwischen einzelnen Sätzen dient.

${ }^{8}$ Gelegentlich führen die Pronomen der 3. Person auch eine Person als Redegegenstand ein (deiktischer Gebrauch): Kennst du sie dort hinten? (Duden 2016: 264). 
Obwohl im Beispiel (3) keine Einzelperson erwähnt wird, nimmt der Hörer die (an ihn gerichtete) Mitteilung wahr und verfolgt die mitgeteilte Aufforderung. Das Merkmal Person wird normalerweise morphologisch am Verb angezeigt. Demgemäß besteht im Beispiel (3) zwischen 2. Person im Singular und 2. Person im Plural ein Unterschied durch Anwesenheit und nicht-Anwesenheit der Verbendungen (iss-Ø/iss-t, schreib-Ø/schreib-t):
(4) 1. schreib-e
Singular
2. schreib-st
3. schreib-t

Die Endungen -e, -st und -t in (4) bzw. -en, -t, -en in (5) sind Portmanteaumorpheme, die außer anderen Merkmalen (Tempus, Modus, Genus Verbi) auch die Merkmale Person und Numerus enthalten. ${ }^{9}$
(5) 1. schreib-en
Plural
2. schreib-t
3. schreib-en

Die Endungen der 1. und 3. Person Plural einerseits und die Endungen der 3. Person Singular und 2. Person Plural anderseits in den Beispielen (4) und (5) sind jeweils identisch. Wegen diesem Synkretismus sind Endungen nicht immer in der Lage eine Personenaufhellung anzudeuten.

Aus der Beobachtung, dass das Merkmal Person in vielen Sprachen sich überwiegend im finiten Verb ausdrückt, wird dann daraus geschlossen, dass es sich auch in semantischer Hinsicht um ein primär verbales Merkmal handelt. ${ }^{10}$ Zudem kennt man keine mit einem Verb versehene Sprache, die die Personenunterschiede nicht auf die eine oder andere Weise an den Verbformen bemerkbar machen. Aus diesem Grunde schlussfolgert Benveniste, dass die Kategorie Person zu den grundsätzlichen und notwendigen Begriffen des Verbs gehört. ${ }^{11}$ Daraus folgend entsteht nicht selten der Eindruck, dass Person ein Merkmal ist, das von einem Verb oder einer Verbalphrase bestimmt wird und (sofern man keine Personalpronomen berücksichtigt) deshalb auch nur am Verb vorkommt. Die sprachgeschichtlichen Untersuchungen zur Entstehung der Person am Verb zeigen allerdings, dass sich die verbalen Endungen der Person aus kommunikativ notwendiger Wiederaufnahme des Subjekts, ehemals als selbstständige, später dann zu postverbalen Enklitika gewordene Personalpronomen entwickelt haben. ${ }^{12}$

\footnotetext{
${ }^{9}$ Eisenberg (2013: 196).

${ }^{10}$ Helbig / Buscha (1996: 28): „Eine Kategorie des Verbs ist die der Person (im grammatischen Sinne)“.

${ }^{11}$ Benveniste (1974: 254).

12 Gehling (2004: 32) und Szczepaniak (2009: 119).
} 
Daraufhin wird Person vielmehr als Merkmal eines Gegenstandes (Gegenstände) verstanden, das durch Nomen oder Pronomen sprachlich repräsentiert werden kann und demzufolge als deren morphologischer Reflex in den Pers./ Num. des Portmanteaumorphems des Verbs erscheint. ${ }^{13}$ Darüber hinaus ist nicht nur die Kategorie Numerus, wie schon oben durch Beispiel (2) angedeutet wurde, sondern auch Person kein semantisches (inhärentes) Merkmal des Verbs. ${ }^{14}$ Infolgedessen ist Person ein kontextuelles Merkmal des Verbs und ein inhärentes semantisches Merkmal des Nomens. Letztendlich ist die Beteiligung einer Person am Sprechakt kein Geschehen oder Sein. Person und Numerus lassen lediglich erkennen, ob das Geschehen vom Sprecher (Sprechern), Hörer (Angesprochenen) oder von dem Besprochenen getragen wird. ${ }^{15} \mathrm{Zu}$ beachten ist dennoch, dass Person und Numerus intrinsisch anders sind: die Person vermittelt extrasyntaktische Informationen über die Teilnehmer des Sprechakts.

\section{Die Kategorie Person und Nominalphrasen}

Bei Nominalphrasen mit Pronomen als Kern der Phrase kommen nach Duden (2016: 816) nur Merkmale der 1. und der 2. Person vor. Alle übrigen Nominalphrasen haben das Merkmal der 3. Person. Formal zeigt sich das in den syntaktischen Relationsverfahren (Kongruenz/ Rektion) zwischen Subjekt und finitem Verb. Mit einem solchen Befund stimmen im Prinzip alle Grammatiken überein. ${ }^{16}$

Da alle Nominalphrasen mit Nomen als Kern der Phrase das Merkmal der 3. Person haben, die durch Personenendungen am Verb bestätigt werden können, wird vorgegeben, dass jedes Nomen im Deutschen für die 3. Person schon im Lexikon fixiert ist. ${ }^{17}$ Olsen (1991: 40) bekräftigt diese Vermutung durch die Tatsache, dass auch das Genus nirgendwo am Nomen selbst in seiner Zitierform/im Nom. Sg. morphologisch ausgedrückt ist und immerhin als ein inhärentes lexikalisches Merkmal des Nomens betrachtet wird. ${ }^{18}$

${ }^{13}$ Darüber hinaus verweise ich auf Heidolph et al. (1981: 540): ,was wir (Person des) Subjekt(s) nennen, ist eher eine semantische Expansion eines Morphems am Verb“".

${ }^{14}$ Merkmale sind Eigenschaften oder Beziehungen, die durch Verben, Adjektive, Nomen (die über kein semantisches Merkmal [+spezifisch] verfügen) bzw. durch Prädikate sprachlich repräsentiert sind.

${ }^{15}$ Heidolph et al. (Ebd.: 540).

${ }^{16}$ Heidolph et al. (1981: 568-569), Helbig / Buscha (1996: 29), Zifonun et al. (1997: 1090), Eisenberg (2013: 286).

17 Die Vermutung, dass Nomen über das Merkmal 3. Person verfügen ist in Gleichstimmung mit der Struktur in (1), wobei Nomen (die ein Ding bezeichnen) zur Besprochenen bzw. NichtTeilnehmer Personen gehören.

18 So etwa auch Heidolph et al. (1981: 268): „Mit dem einzelnen Sb, das als Element des Wortschatzes in die syntaktische Struktur eintritt, liegt außer der Person (3. Person für alle Substantiva) für jedes einzelne $\mathrm{Sb}$ auch das Genus fest“. Siehe aber: dem Manne, des Mannes: der Frau. 
(6) (die) Maus, (das) Haus, (der) Schmaus

Nach Olsen (1996) und Löbel (1996) spielt deshalb das Merkmal Person eine bedeutende Rolle in der Struktur von Nominalphrasen. Das zeigt sich besonders bei Nominalphrasen, die ein Possessivum enthalten.

\section{(7) meinem Wagen}

In Beispiel (7) bezeichnet meinem den Sprecher, daher trägt meinem das Merkmal 1. Person, während das Merkmal für das Nomen Wagen (so Olsen und Löbel) schon im Lexikon als 3. Person vorgegeben ist. Wäre das Possessivum ein DET (Determinant) wie es die traditionelle Auffassung der Nominalphrase annimmt), dann würden unter DET zwei verschiedene Werte des Merkmals Person (1. und 3. Person) aufeinander treffen, was (für beide Autorinnen) keinen Sinn ergibt. Um diesen grammatischen Merkmalswiderspruch in der Nominalphrase zu verweigern, betrachten die Autorinnen Possessiva nicht als ein DET, sondern als Pronomina (Olsen) oder als Adjektive (Löbel). ${ }^{19}$ So z.B. zerlegt Olsen zu diesem Zweck das Possessivum in zwei Teile: einem Pronomen (mein) und einem Poss-Merkmal $(-\mathrm{em})$ bzw. meinem $=$ mein $+\mathrm{em}$. Darüber hinaus nimmt Olsen mein als eine intransitive DP (Pronomen) an, die unabhängig vom Matrix-DET ein Merkmal 1. Person unter dem eigenen Kern der Phrase trägt und dem Poss-Merkmal -em unter der Matrix DET, der das vorbelegte Merkmal 3. Person am Matrix-Knoten genau wie das Nomen Wagen trägt. In der aufgezeigten Weise geht Olsen mit dem Merkmalswiderspruch um.

Nun stellt sich somit die Frage: Ist die 3. Person das einzige Merkmal Person bei Nomen bzw. ist das Merkmal 3. Person schon im Lexikon ausnahmslos für alle Nomen eingetragen? Sie lautet anders formuliert: Könnte man auch annehmen, da es für Nomina keine morphologischen Kennzeichen für das Merkmal Person gibt, dass das Merkmal Person kein (schon eingetragenes) Lexikon-Merkmal des Nomens ist und daher auch nicht zu sein braucht? Es könnte somit ebenso gut sein, dass das Nomen indifferent gegenüber dem Merkmal Person ist und dass das Merkmal Person des Nomens kontextuell bestimmt wird. Um die Antwort auf diese Fragen zu finden, betrachten wir zunächst folgende Beispiele:

(8) a) Anna (du)! Komm-Ø her! Kinder (Ihr)! Komm-t her!

b) Junge (du)! Komm- Ø her! *Der Junge! Komm- Ø her!

Die Ausdrücke in Beispiel (8a) beziehen sich auf die Angeredeten, die sprachlich durch Nomen im Anredenominativ repräsentiert sind. Man sagt, dass diese Nomen über einen Vokativstatus verfügen, aber (formal) keine Vokative sind. Im Unterschied zu einigen anderen Sprachen gibt es im Deutschen keinen Vokativ

${ }^{19}$ Siehe Olsen (1991: 51). 
als morphologische Kasusausprägung. ${ }^{20}$ Die heute gebräuchlichen Anredeformen sind syntaktisch nicht in den Satz integriert, deshalb werden sie in initialer Satzposition mit einem Interpunktionszeichen vom Rest des Satzes getrennt. Charakteristisch für Anredeformen (Satzäquivalente im Nominativ ${ }^{21}$ ) ist auch die entsprechende Intonationskontur, die Mittel - und Endstellung ermöglicht. ${ }^{22}$

(9) Anna (sie) komm-t. Die Kinder (sie) komm-en.

Der Unterschied zwischen den Beispielen in (9) und (8a) besteht darin, dass Anna und Kinder in (8a) nicht jeweils durch das Personenpronomen sie (3. Ps. Singular und 3. Ps. Plural) ersetzt werden können, wie in (9), sondern nur durch $d u$ (2. Ps. Singular) und $i h r$ (2. Ps. Plural). Ein anderer Unterschied ist an den Endungen der Form des Verbs zu erkennen: $-\mathbf{t}$ und $-\mathbf{e n}$ in (9), - $\boldsymbol{\varnothing}$ und -t in (8a). In beiden Fällen verfolgen aber die Endungen das Merkmal ihrer Antezendenten: 3. Person in (9) und 2. Person in (8a). Es sieht so aus, dass Nomen tatsächlich das Merkmal 2. Person ebenso gut tragen können wie das Merkmal 3. Person. ${ }^{23}$

Diese Tatsache ist keine neue Entdeckung, fand aber weniger Beachtung bei den (oben genannten) deskriptiven Grammatiken und gerät fast in Vergessenheit. ${ }^{24}$ Schon von Priscianus (und vor ihm Apollonius Dyscolos) wurde erkannt, dass sich das Merkmal Person nicht nur am Verb zeige, sondern dass jedes im Vokativ erscheinende Nomen das Merkmal 2. Person trägt. ${ }^{25}$ Für das Deutsche hat diese Sichtweise vorerst durch einige sprachwissenschaftliche Beiträge, wie z.B. Herbermann (1994), Beachtung gefunden.

Ursprünglich erkannte Priscianus, das die Nomen alle drei Personen ${ }^{26}$ besitzen. Herbermann (1994: 127) behauptet, dass die deutschen Nomen auch das Merkmal 1. Person tragen können.

(10) Wir Berliner haben/Ihr Berliner habt/Die Berliner haben das Herz auf dem rechten Fleck.

Die Verbindung eines Personalpronomens (1./ 2. oder 3. Person) mit seinem begleitenden Nomen macht nach Herbermann insgesamt eine einzige Nominalphrase aus. Dabei nimmt die gesamte NP jeweils den Personenstatus des betreffenden definiten Pronomens an. Das zeigt sich an den Verbendungen: -en, -t und -en in (10). Herbermann schließt die Möglichkeit aus, das die begleitenden

${ }^{20}$ Zifonun et al. (1997: 28).

${ }^{21}$ DUDEN (2009: 1248).

22 Zifonun et al. (Ebd.: 917).

${ }^{23}$ Natürlich erhebt sich an dieser Stelle allerdings die Frage, was ist grundlegend/ typisch, weshalb trägt nicht auch der Vokativ das Merkmal Person.

24 In diesem Sinne siehe den Apell von Fink (1972).

${ }^{25}$ Siehe Gehling $(2004: 5,30)$ aber auch Fink (1972: 61) und die dazugehörige Literatur.

${ }^{26}$ Fink (Ebd.: 61). 
Nomen in einer Appositionsbeziehung zu den jeweiligen Pronomen im Beispiel (10) steht. Diese Beziehungen sind nach Herbermann nicht nur intonationsmäßig, sondern auch semanto-syntaktisch bzw. kommunikativ verschieden. Darauf folgend vermutet Herbermann für das Nomen Berliner kein festgelegtes Merkmal 3. Person im Lexikon, sondern es deutet vielmehr daraufhin, dass das Nomen das Merkmal Person von dem Kern der Nominalphrase bzw. von dem Pronomen wir übernimmt. ${ }^{27}$

Olsen (1991: 37) befasst sich ebenfalls mit ähnlichen Beispielen:

\section{(11) wir Männer}

Nach Olsen liefern solche Konstruktionen kein so eindeutiges Indiz, dass es sich um eine Appositionsbeziehung handelt. Der Grund für eine solche Unklarheit liegt in der Tatsache, dass hinzutretende Adjektive stark im Singular flektieren:

(12) ich Idiot

ich armer Idiot.

du hartnäckiger Idiot

was die Annahme einer komplexen DP unterstützen würde: Eine intransitive (pronominale) DP (ich/du) plus einen appositiven Bestandteil (Idiot/ armer Idiot/ hartnäckiger Idiot). Im Plural flektiert das Adjektiv aber schwach:

\section{(13) wir armen Männer ihr törichten Linguisten}

was daher eher für eine einfache Phrase spricht. Den ähnlichen Schluss zieht auch Bhatt (1990: 155), die (wie Olsen) keine klare Antwort über den Status solcher Konstruktionen hat.

Es ist bemerkenswert, dass Olsen über die Möglichkeit, dass in (13) zwei verschiedene Werte des Merkmals Person aufeinander treften, schweigsam bleibt, obwohl derselbe Ablauf im Beispiel (7) für das Possessivum unangemessen war.

Wenn wir einerseits die Beispiele mit Possessivum und Konstruktionen mit der Personalnomina 1. und 2. Person vergleichen und dabei annehmen, dass das Merkmal 3. Person des Nomens (Wagen/ Berliner) schon im Lexikon vorgegeben ist:
(14) Meinen Wagen
[1. Person +3 . Person $]=[3$. Person $]$
(15) Wir Berliner
$[1$. Person +3 . Person $]=[1$. Person $]$

${ }^{27}$ Natürlich handelt es sich in diesem Fall um eine NP Struktur, die in Begrifflichkeit von Herbermann dargelegt ist. 
die durch Verbendungen in (16) bzw. (17) festgestellt werden können:

(16) Ich unterzog meinen Wagen einer Reparatur.

(17) Wir Berliner haben das Herz auf dem rechten Fleck.

Die ganze Konstruktion mit Possessivum bzw. NP mit Possessivum (14) trägt (wie das Nomen) das Merkmal 3. Person, während die NP mit Personalpronomen (15) (wie das Personalpronomen) das Merkmal 1. Person trägt.

Was auch immer für ein struktureller Unterschied zwischen (14) und (15) besteht - die resultierenden Werte des Merkmals Person sind in beiden Konstruktionen unterschiedlich, obwohl in diesen Beispielen beide Nomen auf die 3. Person fixiert sind und die jeweiligen begleitenden NP Konstituenten über das Merkmal 1. Person verfügen. Mit anderen Worten, dieselbe Kombination [1. Person +3 . Person] taucht einmal als [3. Person] und ein andersmal als [1. Person] auf. Dies ist offensichtlich ein Indiz dafür, dass das Merkmal Person bei Nominalphrasen von der syntaktischen internen Struktur der Phrase abhängt. ${ }^{28}$ Folglich sollte die DUDEN Behauptung: Alle übrigen Nominalphrasen (außer denen, die ein Personalpronomen der 1. und der 2. Person als Kern beinhalten), haben das Merkmal 3. Person modifiziert werden, entsprechend dem obigen Beispiel (8), wo gezeigt wurde, dass Nomen auch das Merkmal 2. Person tragen können.

\section{Kongruenz oder Korrespondenz?}

Das Merkmal Person ist ein grammatisches Merkmal des Nomens. Obwohl das Nomen über einen spezifischen Wert für das Merkmal Person verfügt, hat aber dafür keine spezifische morphologische Markierung. Gewöhnlich wird dieses NP Merkmal am Verb gekennzeichnet. Nun wollen wir uns genau anschauen, wie das Merkmalübertragungsverfahren von der NP an das Verb weitergeleitet wird. Traditionell wird angenommen, dass das Subjekt und finite Verb in Person und Numerus kongruieren. Die Person-Kongruenz kann durch (18a), während die Numerus-Kongruenz durch (18b) demonstriert werden. ${ }^{29}$

${ }^{28}$ Als zusätzliches Argument dafür, dass die Person vom Kontext abhängig ist, spricht auch die Übernahme der Hörerperspektive, die z.B. im Gespräch mit kleinen Kindern auftritt, wobei nicht selten Verwandtschaftsbezeichnungen zur Selbstbezeichnung gebraucht werden: Oma hat euch was Schönes mitgebracht. In diesem Fall ist Oma Sprecherin, d.h. 1. Person (vgl. Zifonun et al. [1997: 938]. Da sich im Deutschen die grammatische Kongruenz stärker auswirkt als die Synesis [bedeutungsmäBige oder semantische Übereinstimmung], zeigt sich im obigen Beispiel das Merkmal 3. Person am Verb (vgl. Helbig / Buscha [1996: 29]).

${ }^{29}$ Eisenberg (2013b: 286). 
(18) a) ich geh-e du geh-st er geh-t $\mathbf{t}^{30}$

b) ich geh-e wir geh-en

Durch Verbendungen -e, -st und -t in (18a) lassen sich alle drei Personen (im Singular) identifizieren, während durch Verbendungen -e und -en in (18b) der Unterschied zwischen Singular und Plural auffällig wird.

Nicht alle Autoren sind sich jedoch einig, ob zwischen Subjekt und dem finiten Verb eine Kongruenz besteht. So beteuert Eisenberg (2013b: 286) „Die sogenannte Subjekt-Prädikat-Kongruenz ist im Deutschen in den allermeisten Fällen nicht eine reine Kongruenzbeziehung, sondern teilweise oder ganz eine Rektionsbeziehung $[\ldots . .]^{\text {“. }}$. Mit anderen Worten besteht zwischen Subjekt und Prädikat eine formale Korrespondenz. ${ }^{31}$ Seine Vermutung bezieht sich genau auf das Merkmal Person:

(19) Der Mann steht am Tor.

Nach Eisenberg wird im Beispiel (19) der Mann im Kasus und Numerus flektiert, nicht aber bezüglich der Person ,es gibt weder eine 1. noch eine 2. Person zu der Mann [...] ein substantivisches Subjekt fordert für das Verb die 3. Person, eine Paradigmenkategorie regiert eine Einheitenkategorie" und die Wahl 3. Person ist kein Zufall: „Sie beruht darauf, dass man sich auf solche Subjekte mit Pronomina 3. Person bezieht (der Mann (er) )“. ${ }^{32}$ In Hinsicht auf den Numerus dagegen liegt Kongruenz vor, denn das Substantiv flektiert sich ebenfalls im Numerus. Nicht immer kommt jedoch bezüglich der Kategorie Person (so führt Eisenberg weiter) eine Rektionsbeziehung vor. Im Fall, wenn das Subjekt durch Personalpronomina vertreten ist, nimmt Eisenberg an, dass die Kongruenz zwischen Subjekt und finites Verb gegeben ist. ${ }^{33}$ Eisenberg stimmt der DUDEN (2016: 1004) Feststellung nicht zu, dass „das finite Verb mit dem Subjekt in Person und Numerus überein-

${ }^{30}$ In seinem typologischen Ansatz vermutet Lyons (1999: 317), dass -t nur Singular darstellt und keine 3. Person im Singular. Diese Vermutung bekräftigt er mit der typologischen Schlussfolgerung, dass in den meisten Sprachen die 3. Person Singular gewöhnlich unmarkiert ist (-Ø). Das steht ebenfalls im Einklang mit der traditionellen Erfassung der 3. Person. Wie oben schon gezeigt wurde, besteht zwischen 3. und 1./ 2. Person ein wesentlicher struktureller Unterschied: die beiden letzteren beziehen sich auf Teilnehmer der Sprechsituation, die dritte Person dagegen nicht.

${ }^{31}$ Der Terminus Korrespondenz umfasst beide syntagmatische Relationen: Kongruenz und Rektion (vgl. Zifonun et al. 1997: 26).

${ }^{32}$ Unter substantivischem Subjekt versteht Eisenberg eine Nominalphrase, bei der ein Substantiv als Kern der Phrase auftritt.

33 D.h. auch mit der 3. Person. Eisenberg formuliert keine Ausnahmen. 
stimmt“, da „damit wird der Eindruck erweckt, als handele es sich um identische Kategorien".

Für Zifonun et al. (1997: 84) wird die Person am Verb vom Subjekt regiert ungeachtet davon, ob das Subjekt ein Pronomen oder Nomen als Kern (der Phrase) ist. Mit anderen Worten besteht zwischen dem Subjekt und Verb eine syntaktische Relationskorrespondenz (Rektion der Person, Kongruenz im Numerus). ${ }^{34}$ Sowohl bei Eisenberg als auch bei Zifonun wird implizit angenommen, dass das Pronomen und Nomen ihre Werte der Person schon im Lexikon enthalten. Offenbar weist die syntaktische Relationsverfahren-Korrespondenz für Anredeformen bzw. Scheinvokative Schwierigkeiten auf. Diese Schwierigkeiten könnten allerdings behoben werden, falls das Merkmal 2. Person für Anredeformen, die eine spezifische Intonationskontur in der gesprochenen Sprache verfügen und mit einem Interpunktionszeichen in der geschriebenen Sprache erfolgen, angenommen würde. Anders gesagt, jedes Nomen, das über eine Anredeintonationskontur verfügt oder mit einem Interpunktionszeichen erfolgt, sei mit dem Merkmal 2. Person vorgegeben. Eine solche Annahme könnte auch bei dem Kongruenzverfahren behilflich sein. Zu beachten ist aber, dass durch die Abwesenheit des Merkmals Person am Nomen das Kongruenz-Prinzip verletzt wird.

\section{Fazit}

Die Person ist ein grammatisches Merkmal des Nomens, die am Verb bzw. mittels Verbendungen durch syntaktische Relationsverfahren (Kongruenz/ Rektion) weiter geleitet wird. Trotz unsichtbarer Markierung verfügen aber über das (grammatische) Merkmal Person auch einige Konstituenten des nominalen Bereichs (Pronomen, Nomen, Possessivum usw.). Gewöhnlich wird angenommen, dass die Merkmale der 1. und der 2. Person nur bei Nominalphrasen mit Pronomen als Kern vorkommen. Für alle übrigen Nominalphrasen, die als Kern ein Nomen haben, ist das Merkmal 3. Person schon im Lexikon vorgegeben. Es wurde allerdings gezeigt, dass (die übrigen) Nominalphrasen (Anredeformen bzw. Scheinvokative) ebenso gut die 2. Person tragen können. Diese Tatsache kann Schwierigkeiten für die Struktur der Nominalphrase und für das syntaktische Relationsverfahren (Kongruenz/ Rektion) aufweisen. Um diese Schwierigkeiten zu vermeiden wurde Folgendes vorgeschlagen: jedes Nomen, das über eine Anredeintonationskontur verfügt oder mit einem Interpunktionszeichen erfolgt, sollte mit einem vorgegebenen Merkmal 2. Person bezeichnet werden.

34 Vgl. Zifonun et al. (1997: 1683). 


\section{Literatur}

Admoni, Wladimir (1982): Der deutsche Sprachbau. München.

Benveniste, Emil (1974): Probleme der allgemeinen Sprachwissenschaft. München.

Bernstein, Judy (2008): Reformulating the Determiner Phrase Analysis. In: Language and Linguistics Compass 2/6, S. 1246-1270.

Corbett, Grevill (2012): Features. Cambridge.

Duden (2016): Bd. 4. Grammatik. Berlin.

Eisenberg, Peter (2013a): Grundriss der deutschen Grammatik, Bd. 1. Das Wort. Stuttgart.

Eisenberg, Peter (2013b): Grundriss der deutschen Grammatik, Bd. 2. Der Satz. Stuttgart.

Fink, Robert (1997): Person in Nouns: Is the Vocative a Case? In: The American Journal of Philology, Vol. 93, No. 1, Studies in Honor of Henry T. Rowell, S. 61-68.

Gehling, Thomas (2004): Ich, du und andere. Eine sprachtypologische Studie zu den grammatischen Kategorien Person und Numerus. Münster.

Harweg, Roland (1968): Pronomina und Textkonstitution. München.

Herbermann, Glemens-Peter (1994): Die dritte Person. Pronomina und Definitheit. In: Canisius, Peter / Glemens-Herbermann, Peter / Tschauder, Gerhard (Hrsg.): Text und Grammatik. Festschrift für Roland Harweg zum 60. Geburtstag. Bochum. S. 89-129.

Helbig, Gerhard / Joachim, Buscha (1996): Deutsche Grammatik-Ein Handbuch für den Ausländerunterricht. Berlin.

Heidolph, Karl Erich / Flämig, Walter / Motsch, Wolfgang (1981): Grundzüge einer deutschen Grammatik. Berlin.

Jachnow, Helmut et al. (Hrsg.) (1999): Personalität und Person. Slawistische Studienbücher, Neue Folge, Bd. 9.

Löbel, Elisabeth (1996): Kategorisierung der Possessiva als Adjektive in der NP/DP. In: Tappe, Thilo / Elisabeth, Löbel (Hrsg.): Wuppertaler Arbeitspapiere zur Sprachwissenschaft, Bergische Universität Wuppertal. S. 58-94.

Lyons, Christopher (1999): Definiteness. Cambridge.

Lyons, John (1980): Einführung in die moderne Linguistik. München.

Olsen, Susan (1991): Die deutsche Nominalphrase als Determinansphrase. In: Olsen, Susan / Gisbert, Fanselow (Hrsg.): DET, COMP, INFL. Zur Syntax funktionaler Kategorien und grammatischer Funktionen. S. 35-36.

Olsen, Susan (1996): Das Possessivum seine Eigentümlichkeit. In: Tappe, Thilo / Löbel, Elisabeth (Hrsg.): Wuppertaler Arbeitspapiere zur Sprachwissenschaft, Bergische Universität. Wuppertal. S. 35-56.

Siewierska, Anna (2004): Person. Cambridge textbooks in linguistics. Cambridge.

Szczepaniak, Renata (2009): Grammatikalisierung im Deutschen. Tübingen.

Tafel, Karin (1999): Personalität und die Kategorie der Person beim Verb. In: Jachnow, Helmut et al. (Hrsg.): Personalität und Person. Slawistische Studienbücher, Neue Folge, Bd. 9. S. 233-253.

Zifonun, Gisela / Hoffmann, Ludger / Stecker, Bruno (1997): Grammatik der deutschen Sprache. Berlin.

\section{Abstracts}

Obwohl das Merkmal Person zu den grundlegenden grammatischen Merkmalen gehört und der Eindruck besteht, dass alles darüber ausgesagt wurde, sieht es aber aus, dass einige Gegebenheiten noch genauer behandelt werden müssen. Dieser Beitrag untersucht, ob die deutschen Nominalphra- 
sen, die als Kern kein Personalpronomen haben, auch andere Merkmale als das der 3. Person tragen können. Es wird gezeigt, dass Anredeformen nur die 2. Person beanspruchen. Demzufolge wird die (nur) 3. Person Annahme vorgeschlagen, die in allen deskriptiven Grammatiken deutscher Sprache akzeptiert ist, zu modifizieren. Genauer gesagt, sollte das Merkmal 2. Person für jede Nominalphrase vorgegeben werden, die die charakteristische (Vokativ-) Intonationskontur in der gesprochenen Sprache hat oder mit einem bestimmten (Vokativ-) Interpunktionszeichen in der geschriebenen Sprache erfolgt. Somit könnten die Schwierigkeiten bei der Darlegung von Kongruenz/Rektion vermieden werden.

Schlüsselwörter: Person, Nominalphrase, Syntax, Kongruenz, Rektion

\section{Syntactic peculiarities of the feature person in German}

Although the feature Person belongs to the basic grammatical categories and the impression is that everything has been said, it seems, however, that some matters must be treated in more details. Concretely, this article will investigate if German noun phrases which heads are no personal pronouns, could hold other person feature than the 3rd person which is specified in the lexicon. It will be shown that quasi-vocative noun phrases hold 2 nd person only. Consequently, proposal is to modify 3rd person only assumption which is accepted in all descriptive grammars of German language. Specifically, the person feature should be fixed to 2nd person feature for any noun phrase which has a characteristic vocative-intonation contour in the speaking language or is followed with a specific punctuation for vocative noun phrases in written language. In this way all possible problematic issues related to syntactic relations (agreement/government) and noun phrase structure could be avoided.

Keywords: person, nominal phrase, syntax, agreement, government

Teuta Abrashi

Universiteti i Prishtinës

Fakulteti i Filologjisë

Departameti i Gjuhës dhe Letërsisë Gjermane

Rr. „George Busch“ 31

10000 Prishtina

Kosovo

E-Mail: teuta.abrashi@uni-pr.edu 